\title{
Loss of Masticatory Function Affects Morphology of the Tooth Root in Rats
}

\author{
Takamichi Yamada1, Kei Ogawa ${ }^{2,3}$, Takehiko Shimizu ${ }^{2,3}$ \\ ${ }^{1}$ Department of Pediatric Dentistry, Nihon University Graduate School of Dentistry at Matsudo, Chiba, Japan \\ ${ }^{2}$ Department of Pediatric Dentistry, Nihon University School of Dentistry at Matsudo, Chiba, Japan \\ ${ }^{3}$ Nihon University Research Institute of Oral Science, Chiba, Japan \\ Email: mata17014@g.nihon-u.ac.jp
}

How to cite this paper: Yamada, T., Ogawa, K., \& Shimizu, T. (2021) Loss of Masticatory Function Affects Morphology of the Tooth Root in Rats. Open Journal of Stomatology, 11, 37-54.

https://doi.org/10.4236/ojst.2021.111004

Received: December 13, 2020

Accepted: January 25, 2021

Published: January 28, 2021

Copyright (c) 2021 by author(s) and Scientific Research Publishing Inc. This work is licensed under the Creative Commons Attribution International License (CC BY 4.0).

http://creativecommons.org/licenses/by/4.0/

\begin{abstract}
Masticatory hypofunction and soft food affect the tooth rows, occlusion, and jawbone. This study aimed to clarify the influence of tooth loss and a soft diet on morphology of the tooth root during the growth period. We divided 3-weekold Wistar rats into the following three groups: Hard diet group (rats raised on solid standard diet), Powder diet group (rats raised on powdered standard feed diet), and Extraction group (rats raised on powdered standard diet with maxillary molars extraction). Length, width, cross-sectional area, and volume of the root of the mandibular M1 and M2 were measured using micro-CT analysis. Non-decalcified thin-slice specimens of sagittal sections of the M1 were obtained at the age of 20 weeks, and the roots were observed. The root length of all roots in the Extraction group was significantly longer than that in the other groups. The root width and cross-sectional area at the apical side 1/4 of all roots in the Extraction group were significantly smaller than those in the other groups. The root volume of the M1 mesial root in the Extraction group was significantly smaller than that in the other groups. This study clarified that when masticatory stimulus in the immature teeth is reduced by the extraction of opposing teeth and a powder diet, the root length increases due to the promotion of cellular cementum addition at the apex, and the root width and cross-sectional area decrease due to the suppression of cellular cementum addition at the apical side $1 / 4$ of the roots.
\end{abstract}

\section{Keywords}

Rats, Tooth Root Morphology, Powder Diet, Extraction of Molars, Micro-CT

\section{Introduction}

In pediatric dental practice, we often see cases of reduced occlusal function caused 
by early loss of teeth as a result of caries, trauma, or congenital lack of teeth. In recent years, there has been an increase in the production of soft foods, causing children to prefer soft foods over hard foods [1]. It is believed that the tooth rows, occlusion, and mandible are affected by soft food [2] [3] [4].

To date, it has been reported that in rats raised with soft food, masticatory muscle strength decreased [5], and the masticatory muscle function influenced the transversal growth of the skull [6]. Furthermore, reduced occlusal function as a result of tooth extraction reportedly affected the development of the maxillary and mandibular bones [7] [8] [9]. Moreover, regarding the periodontal tissue directly stimulated with an occlusal force, decreased alveolar bone width [10] [11], and narrowed periodontal ligament width [10] [11] [12] have been reported in rats raised on soft feed. On the other hand, decreased alveolar bone height [13], disordered periodontal ligament fibers and fibroblast arrangement [14], narrowed periodontal ligament width [15], and changes in microvasculature of the periodontal ligament [16] were reported in rats whose occlusal function was artificially reduced by opposing teeth extraction, cusp removal, or bite-raising. We reported that a soft diet and tooth loss might worsen the bone microstructure, reduce osteogenesis, and promote bone resorption in the alveolar bone [17]. Additionally, Saeki et al. [18] reported that cementoblast proliferation and cementum addition were observed in the apex of rats whose opposing teeth were extracted. Cementum covers the surface of the root and determines the morphology of the root. The formation of cementum continues throughout the lifetime, and its thickness increases with age; however, its rate of formation is slow [19]. Cementum is classified into acellular and cellular cementum according to the presence or absence of cells. Acellular cementum is distributed on the cervical side $1 / 2-2 / 3$, and cellular cementum is distributed on the apical side $1 / 2-1 / 3$. Several researchers have reported an association between reduced masticatory function and high cementum formation [12] [20] [21]. However, there has been no quantitative study of the effects of changes in food properties and loss of opposing teeth on root morphology.

The purpose of this study is to clarify the relationship between morphology of the tooth root and reduced masticatory function during the growth period. The root length, root width, root cross-sectional area, and root volume of the mandibular first molars (M1) and second molars (M2) were measured and examined using micro $\mathrm{CT}$ in growing rats raised on powder feed and extracted opposing teeth. Furthermore, we observed the roots of the mandibular M1 histologically.

\section{Materials and Methods}

\subsection{Experimental Animals}

We purchased three-week-old Wister rats (21 male rats) from Sankyo Lab Service Co., Ltd., and randomly divided them into the following three groups (seven rats each): 1) Hard diet group: These rats were raised on solid standard feed (MF, Oriental Yeast Co., Ltd, Tokyo, Japan). 2) Powder diet group: These rats 
were raised on powdered standard feed. 3) Extraction group: When the rats were four weeks old, we intraperitoneally administered thiamylal sodium $(15 \mathrm{mg} / \mathrm{kg}$; Kyorin Pharmaceutical Co., Ltd., Tokyo, Japan), and used a spoon excavator under general anesthesia to dislocate and extract all the maxillary molars on both sides; we fed the rats on powdered standard feed. The experimental schedule and procedures are summarized in Figure 1.

The animals were raised in the rat facility at the Laboratory Animal Research Center of our university with a room temperature of $24^{\circ} \mathrm{C} \pm 1{ }^{\circ} \mathrm{C}$, a constant humidity level of $65 \% \pm 5 \%$, and a 12 -hour light/dark cycle. All rats were given distilled water to drink, and had free access to feed and water.

This study was approved by the Ethics Committee of the Nihon University School of Dentistry at Matsudo (animal experiment approval number: AP13MD020). The study design adhered to ARRIVE guidelines, and was approved by the appropriate ethics review board.

\subsection{Measurement of Body Weight}

The body weights of the rats were measured weekly from the age of three weeks until the age of 20 weeks.

\subsection{Micro-CT Analysis}

We imaged the extracted right mandibular body using micro-CT (R_mCT2, Rigaku, Tokyo, Japan). The imaging conditions were as follows: tube voltage, 90 $\mathrm{kV}$; tube current, $160 \mu \mathrm{A}$; magnification, 2x; measurement time, 3 minutes; Field of View, $3 \mathrm{~mm}$. On the cross-section passing through the buccolingual center of the tooth, the tooth root was parallel to the line connecting the mesiodistal CEJ, and was below the line passing through the root furcation (Figure 2(a)). Using 3-D construction analysis software (TRI/3D-BON, Ratoc System Engineering Co, Tokyo, Japan), slice images of the tooth root were constructed in 3-D, and the root length, root width, root cross-sectional area, and root volume were measured for M1 (mesial root and distal root) and M2 (mesiobuccal root, mesiolingual root, and distal root) (Figure 2(b)). Each measurement was performed three times, and the mean of these measurements was used. The measurement

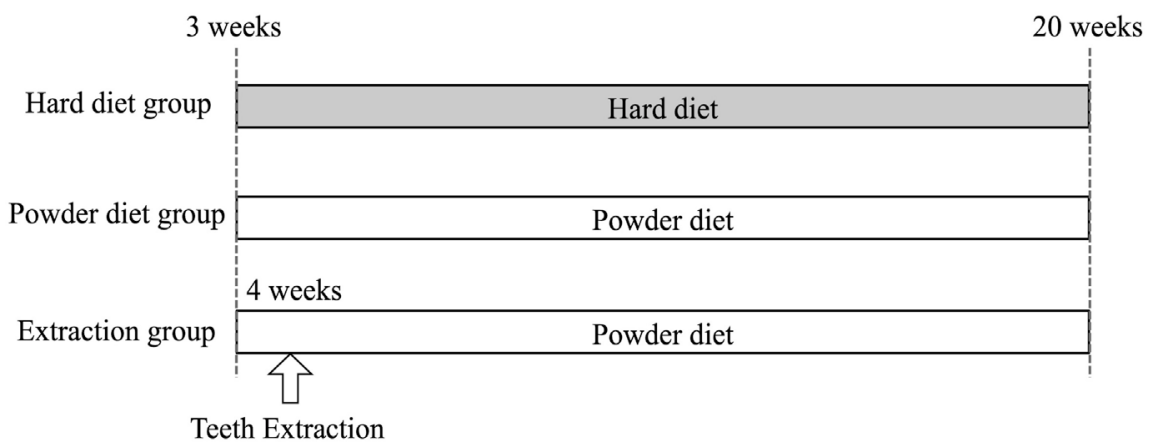

Figure 1. Time-schedule of the experiment. When the rats of the Extraction group were 4 weeks old, we extracted all the maxillary molars on both sides (white arrow). 

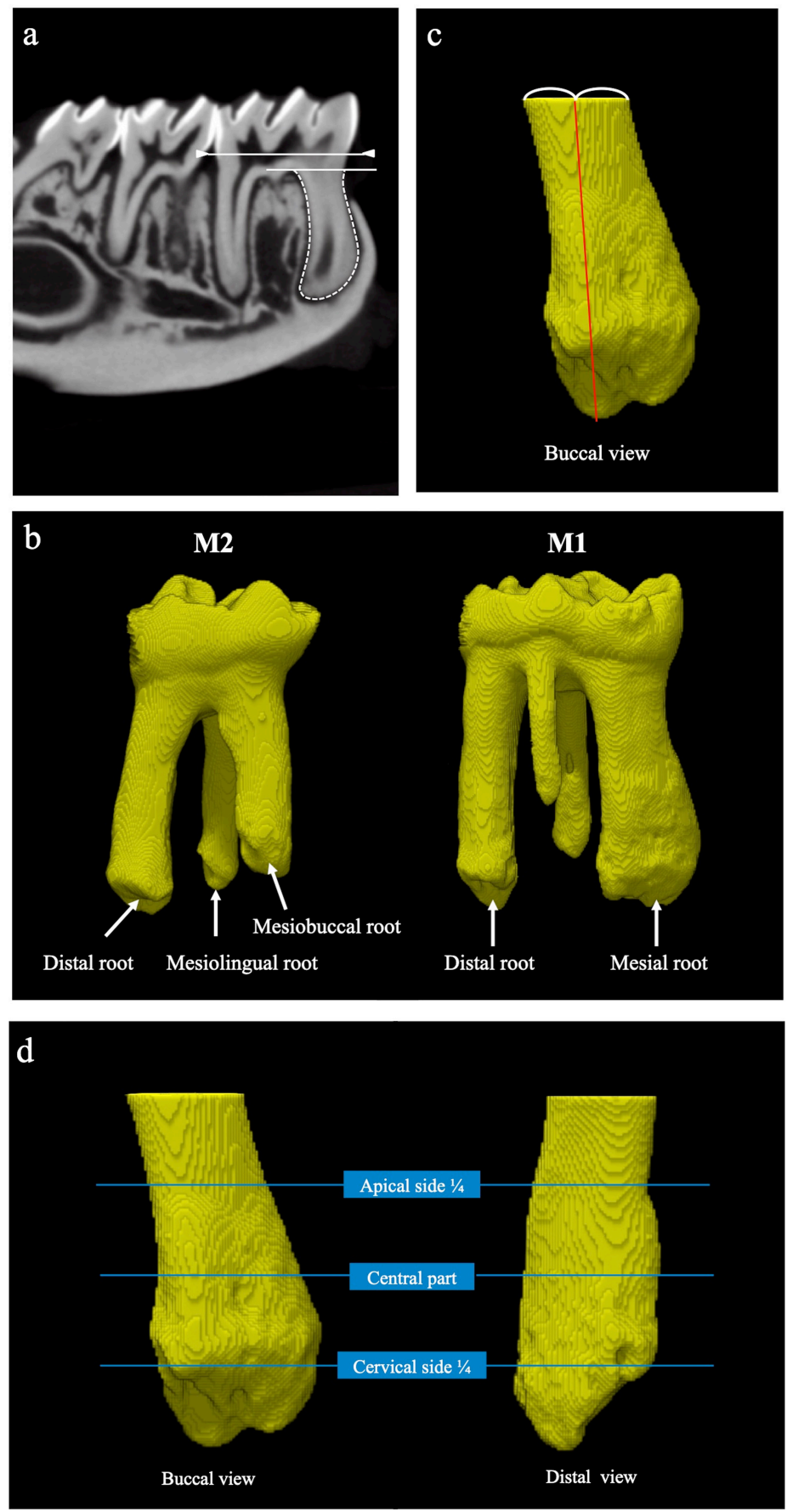

Figure 2. (a) On the cross-section passing through the buccolingual center of the tooth, the tooth root was parallel to the line connecting the mesiodistal CEJ and was below the line passing through the root furcation. (b) The 3-D reconstructed images of M1 (mesial root and distal root) and M2 (mesiobuccal root, mesiolingual root, and distal root). (c) The line connecting the apex from the center of the upper margin of the root was defined as the root length. (d) The mesiodistal root width was measured in buccal view of 3-D images. The buccolingual root width was measured in distal view of 3-D images. The root width and crosssectional area were measured at three places as follows: the cervical side $1 / 4$, central part, and apical side $1 / 4$. 
results were compared among the three groups.

1) Measurement of root length

The line connecting the apex from the center of the upper margin of the root was defined as the root length (Figure 2(c)), and this length was measured in 3-D images.

2) Measurement of root width

The mesiodistal root width was measured in lingual view of 3-D images. The buccolingual root width was measured in distal view of 3-D images. These root width was measured at three places, as follows: the upper 1/4th part of the tooth root on the cervical side (cervical side $1 / 4$ ), the lower $1 / 2$ th part of the tooth root on the apical side (central part) and the lower 1/4th part of the tooth root on the apical side (apical side 1/4) (Figure 2(d)).

3) Measurement of root cross-sectional area

The cross-sectional area of the root was measured at three places in 3-D images as follows: the cervical side 1/4, central part, and apical side 1/4 (Figure $2(\mathrm{~d}))$.

4) Measurement of root volume

The root volume was measured in 3-D images.

\subsection{Statistical Analysis}

All statistical analyses were performed using SPSS (IBM Corp., Armonk, NY). All data are expressed as the mean \pm SD. The ANOVA method was used to compare differences between multiple groups, while differences between individual groups were analyzed by Tukey's test. A p-value less than 0.01 was considered to be statistically significant.

\subsection{Histological Preparation}

We extracted the left mandibular body of each rat, and fixed it in $70 \%$ ethanol. We discarded the soft tissue, and then immersed the bone in Villanueva bone stain for four days, without decalcification. After dehydrating with ethanol, we wrapped the mandibular body with a methyl methacrylate resin. We cut the sagittal sections of the M1 into sections of $5 \mu \mathrm{m}$ thickness using a microtome, and prepared non-decalcified thin-slice specimens for histological observation.

\section{Results}

\subsection{Body Weight}

The mean body weight increased over time for all groups, and no significant differences were found among the groups at any point in time (Figure 3 ).

\subsection{Three-Dimensional Reconstructed Images of the Tooth Root}

The roots of the rats in the Hard diet group and Powder diet group grew laterally around the apical side $1 / 4$, and the apex was rounded. The morphology of the teeth in both groups was similar. On the other hand, the roots of the Extraction 


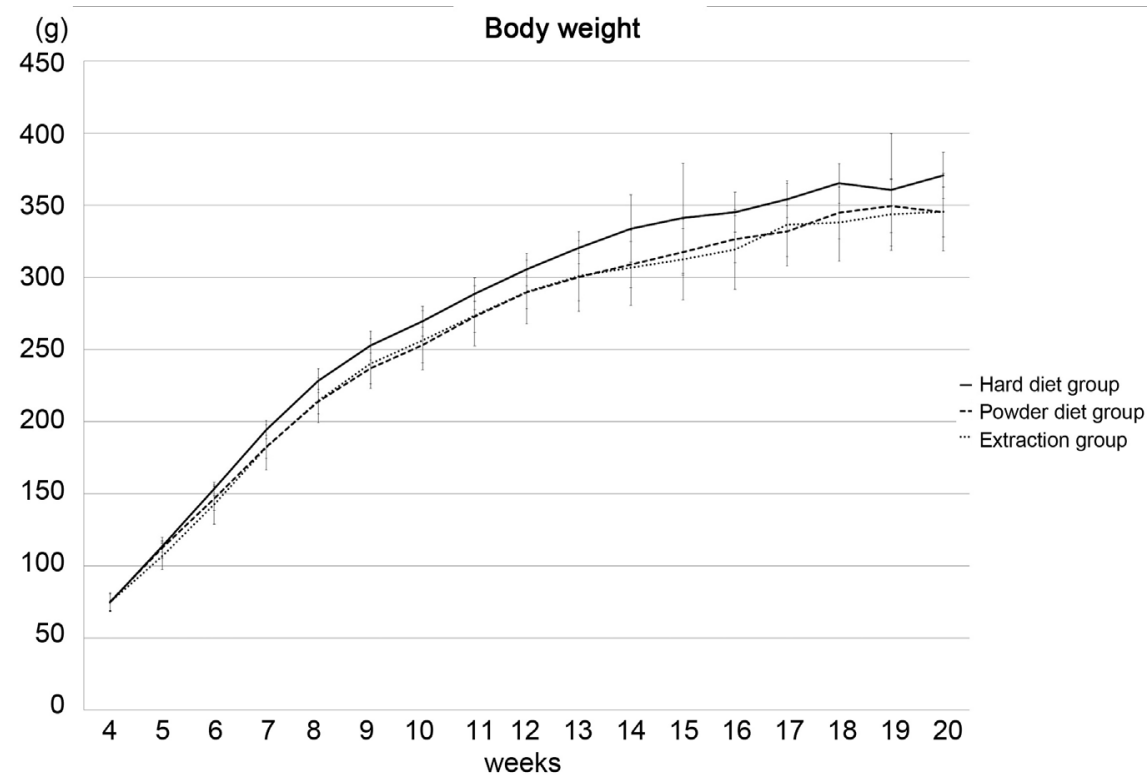

Figure 3. Body weight of the rats, by weeks. The body weight increased with age, and no significant difference was found between the groups at any point in time.

group did not show lateral growth, and the apex was sharp. The root length in the Extraction group was longer than that in the Hard diet group and Powder diet group (Figure 4).

\subsection{Root Length}

Figure 5 shows the results of the measurement of root length. Among the three groups, the Extraction group showed a significantly longer root length than the Hard diet and Powder diet groups. No significant differences were found between the Hard diet group and Powder diet group.

\subsection{Root Width}

Figure 6 shows the results of the measurement of the root width.

1) M1 mesial root

No significant differences were found among the groups in the mesiodistal and buccolingual root widths at the cervical side 1/4. The Extraction group showed significantly thinner mesiodistal and buccolingual root widths at the central part and apical side 1/4, than the Hard diet and Powder diet groups. The Powder diet group showed a significantly thinner mesiodistal root width at the apical side $1 / 4$ than the Hard diet group.

2) M1 distal root

With regards to the mesiodistal and buccolingual root widths at the cervical side 1/4, no significant differences were found among the groups. The Extraction group showed a significantly thinner mesiodistal root width at the central part than the Hard diet group. The Extraction group also showed a significantly thinner mesiodistal root width at the apical side 1/4 than the Hard diet and the Powder diet groups. There were no significant differences among the groups in 


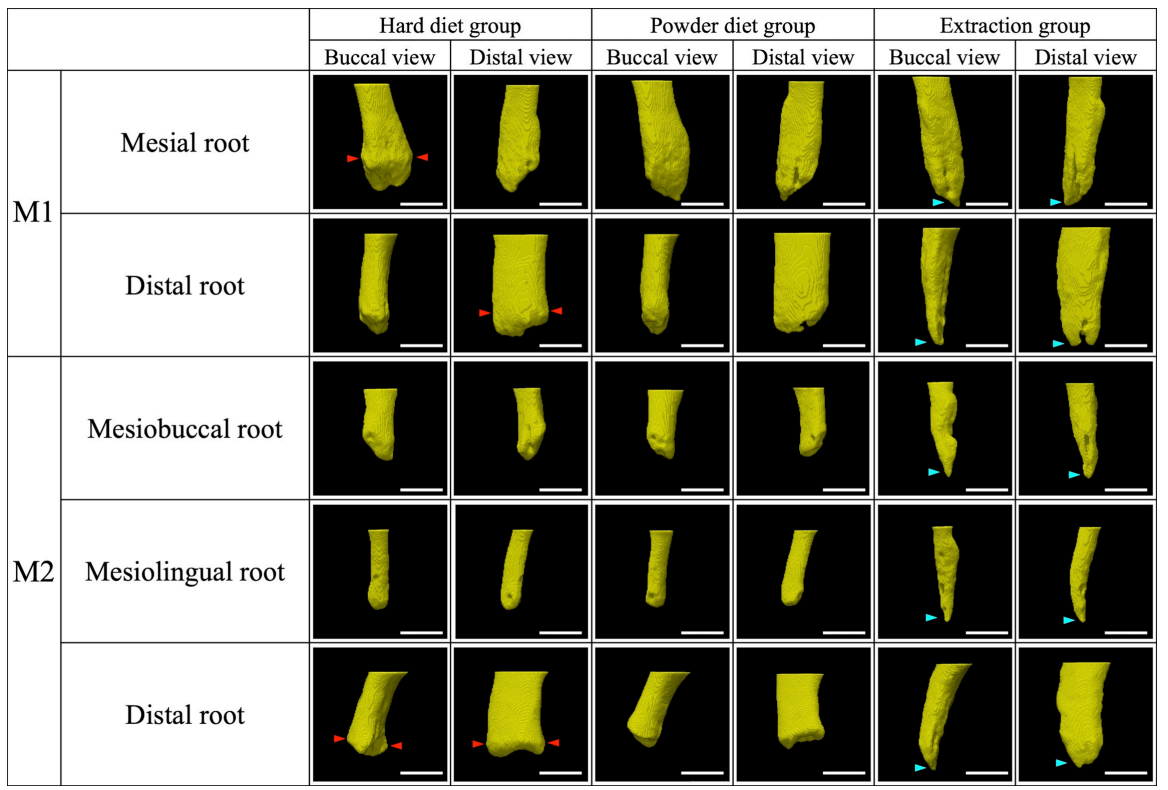

Figure 4. The three-dimensional reconstructed images of the tooth root in buccal and distal views. The roots in the Hard diet group and Powder diet group grew laterally near the apical side 1/4, and the apex was rounded. The Extraction group did not show lateral growth, and the apex was sharp and long. Red arrows indicated lateral thickening of the cementum of the root. Blue arrows indicated sharp point at the apex. Bar: $1000 \mu \mathrm{m}$.
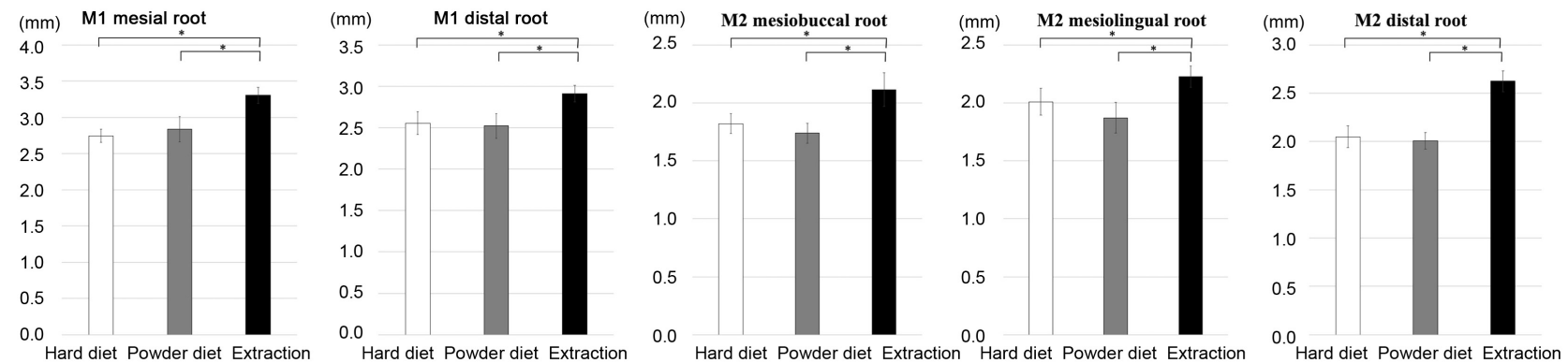

${ }^{*} P<0.01$

Figure 5. Root length in the micro-CT analysis. In all roots, root length in the Extraction group was significantly longer than that in the Hard diet and Powder diet groups. Data are presented as mean \pm standard deviation $\left({ }^{\star} p<0.01\right)$.

the buccolingual root widths at the central part and apical side $1 / 4$.

3) M2 mesiobuccal root

With regards to the mesiodistal and buccolingual root widths at the cervical side 1/4 and central part, no significant differences were found among the groups. However, the Extraction group showed significantly thinner mesiodistal and buccolingual root widths at the apical side 1/4 than the Hard diet and Powder diet groups.

4) M2 mesiolingual root

With regards to the mesiodistal and buccolingual root widths at the cervical side $1 / 4$ and central part, no significant differences were found among the groups. The Extraction group showed significantly thinner mesiodistal and buccolingual root widths at the apical side 1/4 than the Hard diet and Powder 

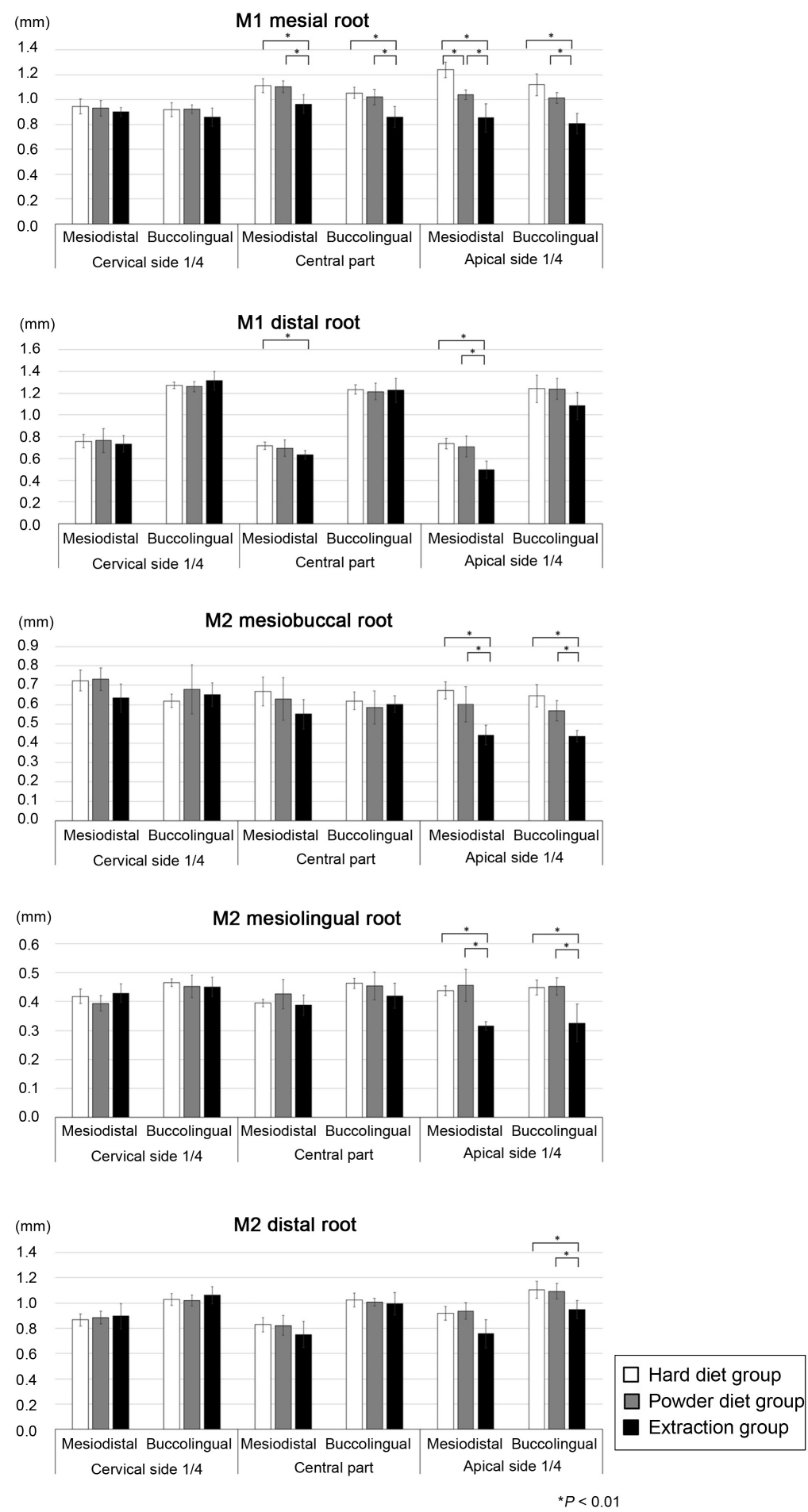

Figure 6. Root width in the micro-CT analysis. At the cervical side $1 / 4$, no significant differences were found among the groups. In the M1 mesial and distal roots at the central part, root width in the Extraction group was significantly smaller than that in the Hard diet group. In all roots at the apical side 1/4, root width in the Extraction group were significantly smaller than those in the Hard diet and Powder diet groups. In the M1 mesial root at the apical side $1 / 4$, the root width in the Powder diet group was significantly smaller than in the Hard diet group. Data are presented as mean \pm standard deviation $\left({ }^{*} p<0.01\right)$. 
diet groups.

5) M2 distal root

With regards to the mesiodistal and buccolingual root widths at the cervical side $1 / 4$ and central part, no significant differences were found among the groups. The Extraction group showed a significantly thinner buccolingual root width at the apical side 1/4 than the Hard diet and Powder diet groups.

\subsection{Root Cross-Sectional Area}

Figure 7 shows the results of the measurement of the root cross-sectional area.

1) M1 mesial root

At the cervical side 1/4, no significant differences were found among the groups. At the central part and apical side 1/4, the cross-sectional area of the Extraction group was significantly smaller than that of the Hard diet and Powder diet groups. At the apical side $1 / 4$, the Powder diet group showed a significantly smaller crosssectional area than the Hard diet group.

2) M1 distal root

At the cervical side 1/4, no significant differences were found among the groups. At the central part, the Extraction group showed a significantly smaller crosssectional area than the Hard diet group. At the apical side 1/4, the Extraction group showed a significantly smaller cross-sectional area than the Hard diet and powder diet groups. At the apical side 1/4, the Powder diet group showed a significantly smaller cross-sectional area than the Hard diet group.

3) M2 mesiobuccal root

At the cervical side $1 / 4$, no significant differences were found among the groups. At the central part, the Extraction group showed a significantly smaller crosssectional area than the Hard diet group. At the apical side 1/4, the Extraction group showed a significantly smaller cross-sectional area than the Hard diet and Powder diet groups.

4) M2 mesiolingual root

At the cervical side 1/4 and central part, no significant differences were found among the groups. At the apical side 1/4, the Extraction group showed a significantly smaller cross-sectional area than the Hard diet and Powder diet groups.

5) M2 distal root

At the cervical side 1/4, no significant differences were found among the groups. At the central part, the Extraction group showed a significantly smaller crosssectional area than the Hard diet group. At the apical side 1/4, the Extraction group showed a significantly smaller cross-sectional area than the Hard diet and Powder diet groups.

\subsection{Root Volume}

Figure 8 shows the results of the measurement of root volume. In the M1 mesial root, the Extraction group showed a significantly smaller root volume than did the Hard diet group. In the other roots, no significant differences were found 

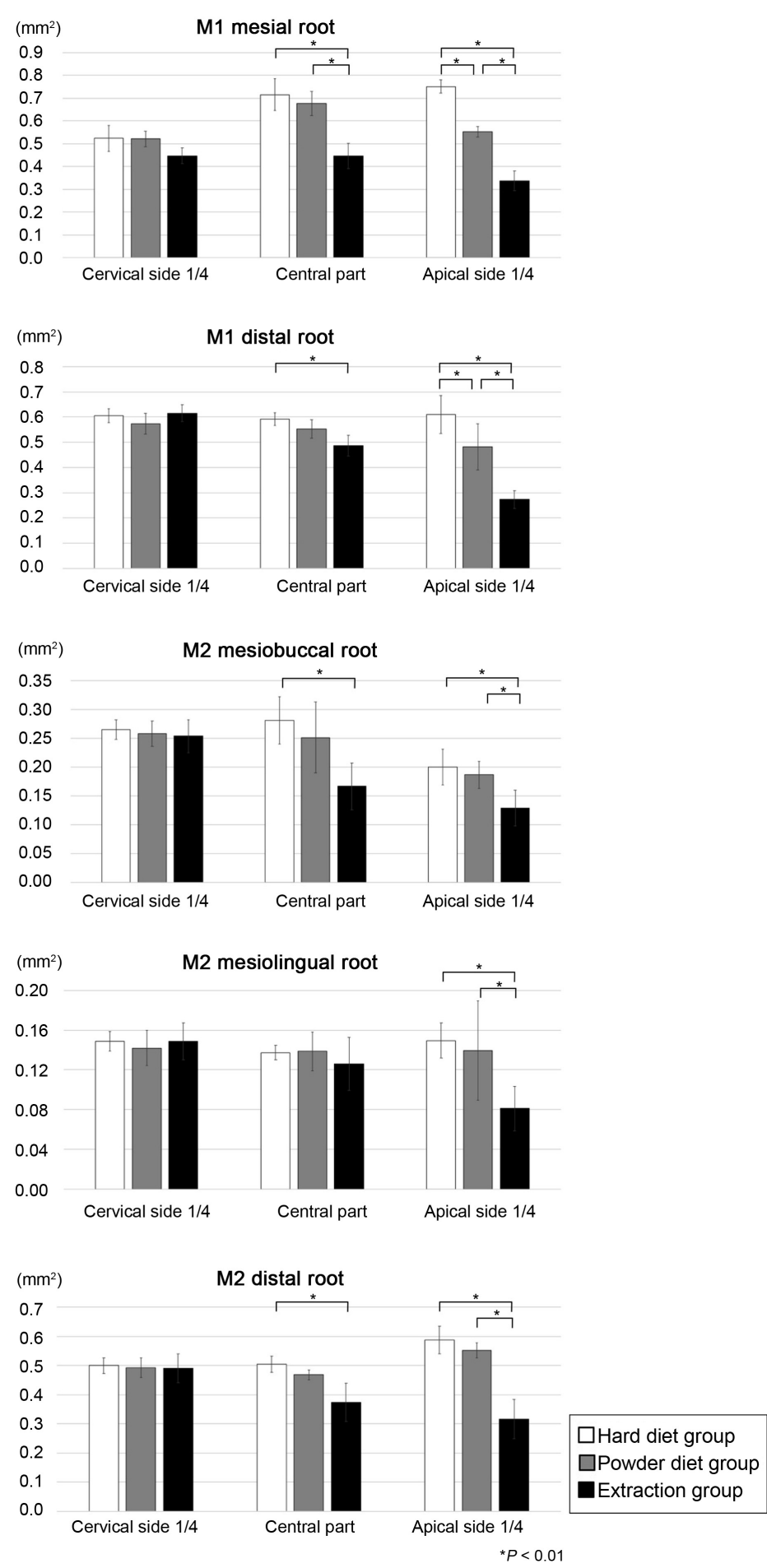

Figure 7. Root cross-sectional area in the micro-CT analysis. At the cervical side $1 / 4$, no significant differences were found among the groups. In all roots at the central part except the M2 mesiolingual root, the cross-sectional area in the Extraction group was significantly smaller than that in the Hard diet group. In all roots at the apical side $1 / 4$, crosssectional area in the Extraction group were significantly smaller than that in the Hard diet and Powder groups. In the M1 root at the apical side 1/4, the cross-sectional area in the Powder diet group was significantly smaller than that in the Hard diet group. Data are presented as mean \pm standard deviation $\left({ }^{*} p<0.01\right)$. 

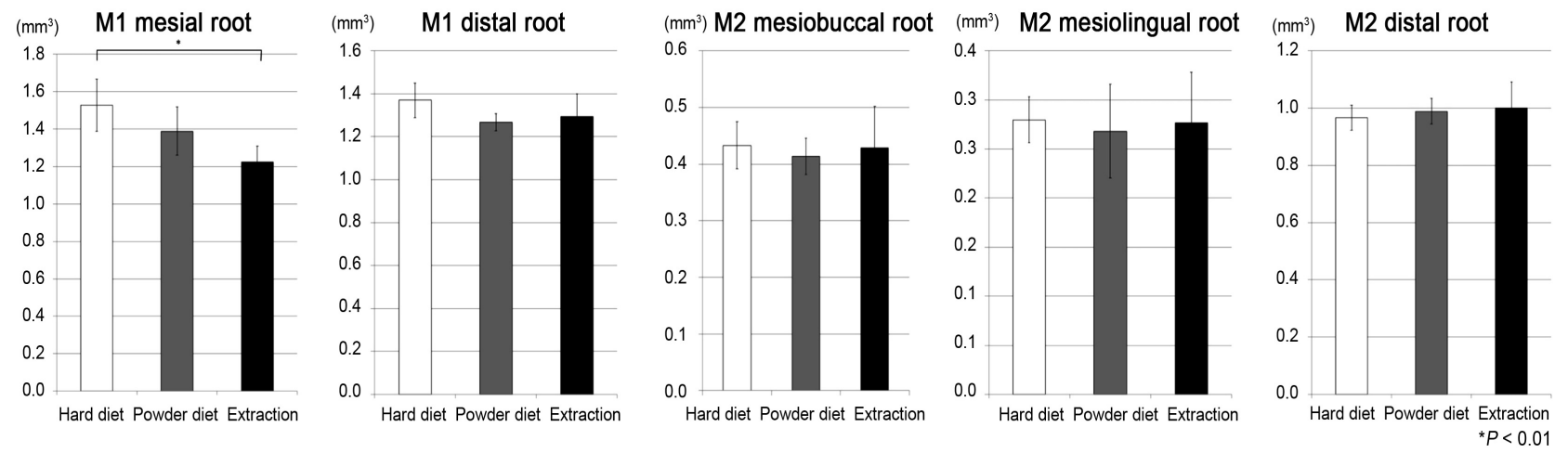

Figure 8. Root volume in the micro-CT analysis. In the M1 mesial root, the root volume in the Extraction group was significantly smaller than that in the Hard diet group. Data are presented as mean \pm standard deviation $\left({ }^{*} p<0.01\right)$.

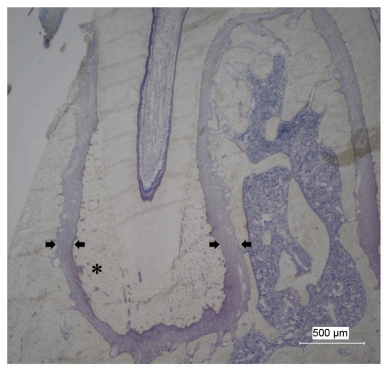

Hard diet group

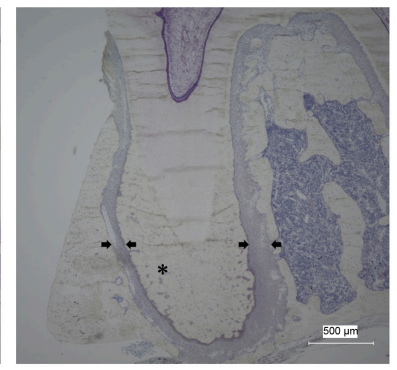

Powder diet group

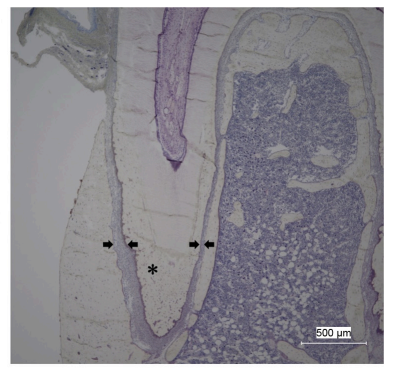

Extraction group

Figure 9. Tissue images of the sagittal sections of the M1 mesial root. Arrows indicate the periodontal membrane width. The asterisk indicates cellular cementum. Hard diet group: Significant lateral thickening of the cementum in the roots was noted. Powder diet group: The cementum thickness was intermediate to that in the Hard diet group and the Extraction group. The periodontal ligament was narrower than the Hard diet group. Extraction group: The cementum thickness on the lateral surface of the apical side $1 / 4$ was thinner, while the cementum thickness at the apex was thicker than in the Hard diet group. The periodontal ligament was the narrowest among the three groups.

among the groups.

\subsection{Histological Observation}

In all groups, the cervical root surface $1 / 2$ was covered with a layer of acellular cementum. On the other hand, the apical root surface $1 / 2$ was covered with cellular cementum. Thickening of the cementum was noted from the cervical $1 / 2$ to the apex. In particular, in the Hard diet group, significant lateral thickening of the cementum in the roots was noted. In the Extraction group, the cementum thickness on the lateral surface of the apical side $1 / 4$ was thinner, while the cementum thickness at the apex was thicker than in the Hard diet group. The cementum thickness in the Powder diet group was intermediate to that in the Hard diet group and the Extraction group. In the morphology of root dentin, no differences were found among the groups. Compared to the Hard diet group, the periodontal ligament was narrower in the Powder diet group, and was the narrowest in the Extraction group (Figure 9). 


\section{Discussion}

The study aimed to clarify the effects of loss of opposing teeth and powder diet on root morphology during the growth period. In the Extraction group, we noted an increase in root length as a result of cellular cementum addition at the apex, and a decrease in root width and cross-sectional area as a result of reduced cellular cementum addition on the lateral surface of the apical side $1 / 4$. As a result of the above, a decrease in volume was noted in the M1 mesial root of the Extraction group. In the Powder diet group, we noted a decrease in the root width and cross-sectional area due to decreased cellular cementum addition on the lateral surface of the apical side $1 / 4$.

We used rats as test subjects because they mature quickly, which allowed us to administer feed to many subjects under controlled conditions. Another reason was that many studies have used rats examine the effects of a soft diet or an antagonist tooth extraction on periodontal tissue. In the Extraction group, we reduced masticatory stimulation to the mandibular alveolar bone even further by extracting the molars (in addition to feeding a powder diet).

Regarding normal root dentin length and cementum, Hoffman and Schour et al. [22] found that in the mandibular first molars of Wistar rats, the thickness of the acellular cementum increases in a direction that is almost parallel to the dentin-cementum boundary; however, this increase slows over time. On the other hand, cellular cementum addition begins on the $35^{\text {th }}$ day after birth, and increases as the length of the root dentin grows. However, after the $12^{\text {th }}$ week after birth, instead of the formation of root dentin, cellular cementum is added at the apex. This addition gradually increases over time. Therefore, in this study, to examine the effects prior to the start of cellular cementum addition, powder feed was given to the Powder diet group and the Extraction group at three weeks of age, and tooth extraction was performed at four weeks of age.

We observed no inter-group differences in body weight of the rats at any point. Body weight increased over time in all three groups. This finding denoted that a powdered diet and tooth loss had no physiological effect on rats. Therefore, physiological conditions can be excluded as a reason for changes in the tooth root morphology observed in the experimental groups.

Root morphology can be observed by extracting the tooth, but at 20 weeks old, the root is longer and the alveolar bone is harder than at four weeks old, so the root is likely to break during tooth extraction. In this study, we used micro CT to separate the root and alveolar bone and construct a three-dimensional image of the root. As such, we were able to evaluate the three-dimensional morphology of the root without tooth extraction. Furthermore, it was easy to measure the length, cross-sectional area, volume, etc., and we think that micro CT was effective.

To quantitatively evaluate the morphology of the tooth root, root width and root cross-sectional area were measured at the cervical side $1 / 4$, central part, and apical side 1/4. Additionally, regarding the root width, mesiodistal and bucco- 
lingual width were measured. To reduce measurement error, these measurements were made three times, and the mean of measurements was used. Since the root morphology of M3 differed individually, root morphology observation and measurement were performed with M1 and M2. In this study, on the cross section passing through the buccolingual center of the tooth, the tooth root was parallel to the line connecting the mesiodistal CEJ and was below the line passing through the root furcation. The root length was defined as the distance from the upper margin of the center to the apex, to avoid the influence of root dilaceration or inclination.

Based on 3-D reconstructed images, the roots in the Hard diet group and Powder diet group grew laterally near the apical side $1 / 4$, and the apex was rounded. On the other hand, the Extraction group did not show lateral growth, and the apex was sharp and long. These findings suggested that the extraction of opposing teeth prevented the lateral growth near the apical side $1 / 4$ and caused the apex to become pointed and longer. Furthermore, the tooth root morphology in the Hard diet group and Powder diet group were similar, suggesting that a powdered diet does not affect root morphology.

In all roots, root length in the Extraction group was significantly longer than that in the Hard diet and Powder diet groups. The length of root dentin could not be measured in this study because separating dentin and cementum was difficult using micro CT. In the tissue images, the amount of cellular cementum at the apex in the Extraction group was significantly more than that in the Hard diet and the Powder diet groups. Levy [23] and Johnson [24] et al. reported that when opposing teeth were extracted, bone formation was noted at the root furcation and between adjacent teeth at an early stage, and that the teeth were extruded. In this study, tooth extraction was performed at four weeks old when the roots were still immature and growing longitudinally. We believe tooth extrusion during this period reduced masticatory pressure in the apex, and reduced the suppression of physiologically applied longitudinal growth. The results suggested that the formation of cellular cementum at the apex in the Extraction group was actively prevalent, and that the root length increased significantly. It has been reported that in human decalcified sections, impacted teeth uninvolved in occlusion show a high degree of cementum thickening, and that thick cementum formation also occurs at the furcation [25]. It has been reported that cementoblast proliferation and cellular cementum addition was noted in rats [18], and we obtained the same results in this study. Furthermore, tooth extrusion was identified as a cause of hypercementosis, which is one of the progressive conditions involving cementum [26]. We believe that the results of our study support this finding. With reference to root length, no significant differences were found between the Hard diet and Powder diet groups. To date, several reports have stated that the alveolar bone mass and bone density decrease when rats are raised on a soft feed [17] [27] [28], and we believe that the Powder diet group experienced a lower masticatory pressure at the apex than did the Hard diet group. However, cementum is a tissue that does not undergo bone-like remode- 
ling, though it changes as dynamically as do bones. The results of this study suggest that the change in masticatory pressure as a result of a powder diet does not affect the formation of cementum at the apex.

With regards to the root width and cross-sectional area at the cervical side 1/4, no significant differences were found among the groups. In the M1 mesial and distal roots at the central part, root width in the Extraction group was significantly smaller than that in the Hard diet group. In all roots at the central part except the M2 mesiolingual root, the cross-sectional area in the Extraction group was significantly smaller than that in the Hard diet group. In all roots at the apical side 1/4, root width and cross-sectional area in the Extraction group were significantly smaller than those in the Hard diet and Powder groups. The root width of the M1 mesial root and the cross-sectional area of the M1 mesial and distal roots at the apical side $1 / 4$ were significantly smaller in the Powder group than in the Hard diet group. In tissue images, no differences in the morphology of root dentin were found among the groups. Furthermore, in contrast to the root thickening caused by cellular cementum addition near the apical side $1 / 4$ in the Hard diet group, cellular cementum addition was smaller in the Extraction group. The extent of root thickening cause by cellular cementum addition in the Powder diet group was intermediate to that in the Hard diet group and the Extraction group. We believe that this caused the difference in root width and cross-sectional area at the central and apical side 1/4 of the root among the three groups. It has been reported that cementum formation is not related to mechanical stimulation, as a high degree of cementum thickening has been observed in unerupted teeth not involved in mastication [25] [26] [29]. On the other hand, it has been reported that cementum thickening observed at the apex or at the furcation is caused by strong mechanical stimulation at this site due to mastication [30]. Furthermore, cellular cementum has also been reported to develop on acellular cementum in response to functional requirements [31]. In this study, compared to the Hard diet group which was given solid feed, the masticatory stimulus was weaker in the experimental groups which were feed a powdered diet or extracted the opposite teeth. Based on histological images, the periodontal ligament width in the lateral side of the root was smaller in the Extraction group than in the Hard diet group. The histological features of a hypofunctional periodontium include a progressive decrease in the number and density of the fibers and their disorientation, and a narrowing of the periodontal ligament [20] [21] [32] [33]. Based on these reports, it can be inferred that the masticatory stimulus on the lateral side of the root is weaker in the Extraction group than in the Hard diet group. Furthermore, it has been reported that the strength of masticatory muscles and the bone density of the alveolar bone decreased in rats which were fed soft food and in those which extracted the teeth [5] [17]. We believe that these may influence a masticatory stimulus on the root. The above mentioned findings suggest that cellular cementum was added on the central and apical side $1 / 4$ of the root in proportion to the degree of the masticatory stimulus acting on the root. Moreover, significant differences in the mesiodistal width and buccolingual width were 
noted between the Hard diet group and the Extraction group. Based on this finding, we believe that the cementum addition, which was a reaction to masticatory stimuli, occurred in the same way in the mesiodistal and buccolingual parts. Compared to the Hard diet group, root width or cross-sectional area of only M1 were smaller in the Powder diet group. The results of this study suggest that the change in masticatory pressure as a result of a powder diet does not affect the formation of cementum at the apical side $1 / 4$ too much. On the other hand, on the cervical side $1 / 4$, the results suggest that the decrease in masticatory stimulation due to a powder diet and the extraction of opposing teeth did not affect the morphology of the root.

It is well known that root resorption occurs when excessive pressure is applied to the root as a result of occlusal trauma or orthodontic treatment. It has been reported that occlusal trauma occurred when a micro-plus-screw pin was inserted into the opposing teeth in mice; additionally, multinuclear giant cells and resorption cavities were also observed on the cementum surface [34]. It has also been reported that excessive orthodontic force induces RANKL-mediated odontoclast differentiation and apoptosis in cementum, resulting in root resorption [35]. From the results of this study, the addition of cellular cementum on the lateral surface of the apical side $1 / 4$ occurs actively under appropriate masticatory pressure. Moreover, our results clarified that when the masticatory pressure decreased, the addition of cellular cementum on the lateral surface of the apical side $1 / 4$ was suppressed. This suggests that cementum is formed according to the mechanical environment, and is adjusted to a shape and mass suitable for maintaining its strength.

In the M1 mesial root, the root volume in the Extraction group was significantly smaller than that in the Hard diet group. Considering the root length, root width, and cross-sectional area, the root length was longer in the Extraction group than in the Hard diet group. However, we believe that the cementum thickening on the lateral surface of the apical side 1/4 of the Hard diet group was more remarkable. Furthermore, the root length of the Powder diet group was almost the same as that of the Hard diet group, and the root width on the lateral surface of the apical side 1/4 did not decrease as much as it did in the Extraction group. On the other hand, no significant differences in the root volume of the other roots were found among the groups. In the Extraction group, the root width on the lateral surface of the apical side $1 / 4$ was smaller than that in the Hard diet group. However, it was supposed that this decrement and increment of the tooth root length were almost equal results. In the Powder diet group, we believe that both the root length and the root width on the lateral surface of the apical side 1/4 were almost the same as in the Hard diet group.

The above findings clarified that when masticatory stimulus in the immature teeth decreases as a result of a powder diet or due to opposing teeth extraction, the root length increases due to the promotion of cellular cementum addition at the apex, and the root width and cross-sectional area decrease due to the suppression of cellular cementum addition on the lateral surface of the apical side 
1/4. These results suggest that the same phenomenon may occur in cases where masticatory stimulation decreases due to early loss of teeth as a result of trauma, dental caries, or congenital deficiency encountered in pediatric dental clinics.

\section{Conclusions}

To summarize, in our study, the Extracion group exhibited longer tooth root and addition of cellular cementum at the apex. Compared to the Hard diet group, the Extracion group showed also smaller tooth width and cross-sectional area.

This study clarified that when masticatory stimulus in the immature teeth is reduced by the extraction of opposing teeth and a powder diet, the root length increases due to the promotion of cellular cementum addition at the apex, and the root width and cross-sectional area decrease due to the suppression of cellular cementum addition at the apical side $1 / 4$ of the roots.

\section{Acknowledgements}

We would like to thank Editage (https://www.editage.com ) for English language editing.

\section{Conflicts of Interest}

There are no conflicts of interest to declare.

\section{References}

[1] Takanashi, N., Naya, K., Masumori, M., Nakano, J., Ohama, A., Hirata, J. and Akasaka, M. (1989) On a Survey on Nutrition of Infants in the Tokyo Metropolitan Area Part I: On Carious Dentitions, Anomalies of Occlusion and Ways of Eating. The Japanese Journal of Pediatric Dentistry, 27, 708-715.

[2] Corruccini, R.S. and Lee, G.T.R. (1984) Occlusal Variation in Chinese Immigrants to the United Kingdom and Their Off-Spring. Archives of Oral Biology, 29, 779-782. https://doi.org/10.1016/0003-9969(84)90006-2

[3] Waugh, L.M. (1937) Influence of Diet on the Jaws and Face of the American Eskimos. The Journal of the American Dental Association and the Dental Cosmos, 24, 1640-1647. https://doi.org/10.14219/jada.archive.1937.0295

[4] Lieberman, D.E., Krovitz, G.E., Yates, F.W., Devlin, M. and Claire, M.S. (2004) Effects of Food Processing on Masticatory Strain and Craniofacial Growth in Retrognathic Face. Journal of Human Evolution, 46, 655-677. https://doi.org/10.1016/j.jhevol.2004.03.005

[5] Kiliaridis, S. and Shyu, B.C. (1988) Isometric Muscle Tension Generated by Masseter Stimulation after Prolonged Alteration of the Consistency of the Diet Fed to Growing Rats. Archives of Oral Biology, 33, 467-472. https://doi.org/10.1016/0003-9969(88)90026-X

[6] Katsaros, C., Berg, R. and Kiliaridis, S. (2002) Influence of Masticatory Muscle Function on Transverse Skull Dimensions in the Growing Rat. Journal of Orofacial Orthopedics, 63, 5-13. https://doi.org/10.1007/s00056-002-9903-0

[7] Da Costa, F.F., Santos, G.S.M.Q., Farias-Neto, A., Sanchez-Ayala, A. and RizzattiBarbosa, C.M. (2015) The Relationship between Occlusal Support and Maxillary Development: An Animal Study. European Journal of Dentistry, 9, 400-403. 
https://doi.org/10.4103/1305-7456.163230

[8] Farias-Neto, A., Martins, A.P. and Rizzatti-Barbosa, C.M. (2012) The Effect of Loss of Occlusal Support on Mandibular Morphology in Growing Rats. Angle Orthodontist, 82, 242-264. https://doi.org/10.2319/060711-373.1

[9] Muramatsu, H., Zhang, X. and Ogawa, K. (2012) Jawbone Morphology in Rats with Extracted Maxillary Molars Reared on Powdered Diet. International Journal of OralMedical Sciences, 11, 211-217. https://doi.org/10.5466/ijoms.11.211

[10] Bresin, A., Johansson, C.B. and Kiliaridis, S. (1994) Effects of Occlusal Strain on the Development of the Dentoalveolar Process in the Growing Rat: A Morphometric Study. European Journal of Experimental Musculoskeletal Research, 3, 112-122.

[11] Denes, B.J., Mavropoulos, A., Bresin, A. and Kiliaridis, S. (2013) Influence of Masticatory Hypofunction on the Alveolar Bone and the Molar Periodontal Ligament Space in the Rat Maxilla. European Journal of Oral Sciences, 121, 532-537. https://doi.org/10.1111/eos.12092

[12] Stahl, S.S. and Derizen, S. (1964) The Adaptation of the Rat Periodontium to Prolonged Feeding of Pellet, Powder and Liquid Diets. The Journal of Periodontology, 35, 312-319. https://doi.org/10.1902/jop.1964.35.4.312

[13] Kinoshita, Y., Tonooka, K. and Chiba, M. (1982) The Effect of Hypofunction on the Mechanical Properties of the Periodontium in the Rat Mandibular First Molar. Archives of Oral Biology, 27, 881-885. https://doi.org/10.1016/0003-9969(82)90045-0

[14] Takaki, Y., Akagawa, Y., Hamada, S., Tamagawa, H., Sato, T. and Tsuru, H. (1978) An Experimental Study on Periodontal Tissue Changes Following Loss of Occlusal Contact. The Journal of the Japan Prosthodontic Society, 22, 759-766. https://doi.org/10.2186/jips.22.759

[15] Motokawa, M., Terao, A., Karadeniz, E.I., Kaku, M., Kawara, T., Matsuda, Y., Gonzales, C., Darendeliler, M.A. and Tanne, K. (2013) Effects of Long-Term Occlusal Hypofunction and Its Recovery on the Morphogenesis of Molar Roots and the Periodontium in Rats. Angle Orthodontist, 83, 597-604. https://doi.org/10.2319/081812-661.1

[16] Suga, H. and Imai, H. (2002) Changes in the Microvasculature of the Rat Molar Periodontal Ligament Due to Loss of Occlusal Function. Shika Lgaku (Journal of the Osaka Odontological Society), 65, 91-105.

[17] Ogawa, K., Yamada, T., Hirai, N., Morita, W., Okada, Y., Yamamoto-Nemoto, S. and Shimizu, T. (2020) Influence of Masticatory Functional Loss on the Remodeling of Alveolar Bone in Rats. Open Journal of Stomatology, 10, 341-359. https://doi.org/10.4236/ojst.2020.1012032

[18] Saeki, M. (1959) Experimental Disuse Atrophy and Its Repairing Process in the Periodontium of Rat Molar. Journal of the Japanese Stomatological Society, 26, 317-347. https://doi.org/10.5357/koubyou.26.317

[19] Formicola, A.J., Krampt, J.I. and Witte, E.T. (1971) Cementogenesis in Developing Rat Molars. Journal of Periodontology, 42, 766-773. https://doi.org/10.1902/jop.1971.42.12.766

[20] Anneroth, G. and Ericsson, S.G. (1967) An Experimental Histological Study of Monkey Teeth without Antagonist. Odontologisk Revy, 18, 345-359.

[21] Pihlstrom, B.L. and Ramfjord, S.P. (1971) Periodontal Effect of Nonfunctional in Monkeys. Journal of Periodontology, 42, 748-756. https://doi.org/10.1902/jop.1971.42.12.748

[22] Hoffman, M.M. and Schour, I. (1941) Quantitative Studies in the Development of the Rat Molar. II. Alveolar Bone, Cementum, and Eruption. American Journal of 
Orthodontics and Oral Surgery, 26, 854-874. https://doi.org/10.1016/S0096-6347(40)90051-5

[23] Levy, G.G. and Mailland, M.L. (1980) Histologic Study of the Effects of Occlusal Hypofunction Following Antagonist Tooth Extraction in the Rat. Journal of Periodontology, 51, 393-399. https://doi.org/10.1902/jop.1980.51.7.393

[24] Johnson, R.B. (1990) Effect of Altered Occlusal Function on Transseptal Ligament and New Bone Thicknesses in the Periodontium of the Rat. American Journal of Anatomy, 187, 91-97. https://doi.org/10.1002/aja.1001870110

[25] Kellner, E. (1931) Influence of Function on the Width of Periodontal Membrane, and Cementum in Regard to Function. Journal of Dental Research, 11, 511-513.

[26] Shafer, W.G., Hine, M.K. and Leby, B.M. (1983) Hypercementosis. In: A Textbook of Oral Pathology, 4th Edition, W. B. Saunders Co., Philadelphia, 333-335.

[27] Bresin, A., Kiliaridis, S. and Strid, K.G. (1999) Effect of Masticatory Function on the Internal Bone Structure in the Mandible of the Growing Rat. European Journal of Oral Sciences, 107, 35-44. https://doi.org/10.1046/j.0909-8836.1999.eos107107.x

[28] Kiliaridis, S., Bresin, A., Holm, J. and Strid, K.G. (1996) Effects of Masticatory Muscle Function on Bone Mass in the Mandible of the Growing Rat. Acta Anatomica, 155, 200-205. https://doi.org/10.1159/000147805

[29] Orban, B.J. (1953) Oral Histology and Embryology. 3rd Edition, C.V. Mosby Co., St. Louis, 154-175.

[30] Häupl, K. (1940) Grundriss der Histo-Pathologie des Zahnes und Seines Stutzapparates. Georg Thieme, Leipzig.

[31] Selvig, K.A. (1964) An Ultrastructural Study of Cementum Formation. Acta Odontologica Scandinavica, 22, 105-120. https://doi.org/10.3109/00016356408993967

[32] Kronfeld, R. (1931) Histological Study of the Influence of Function on the Human Periodontal Membrane. Journal of the American Dental Association, 18, 1242-1274. https://doi.org/10.14219/jada.archive.1931.0191

[33] Cohn, S.A. (1965) Disuse Atrophy of the Periodontium in Mice. Archives of Oral Biology, 10, 909-919. https://doi.org/10.1016/0003-9969(65)90084-1

[34] Fujii, T., Takaya, T., Mimura, H., Osuga, N., Matsuda, S. and Nakano, K. (2014) Experimental Model of Occlusal Trauma in Mouse Periodontal Tissues. Journal of Hard Tissue Biology, 23, 377-380. https://doi.org/10.2485/jhtb.23.377

[35] Minato, Y., Yamaguchi, M., Shimizu, M., Kikuta, J., Hikida, T., Hikida, M., Suemitsu, M., Kuyama, K. and Kasai, K. (2018) Effect of Caspases and RANKL Induced by Heavy Force in Orthodontic Root Resorption. The Korean Journal of Orthodontics, 48, 253-261. https://doi.org/10.4041/kjod.2018.48.4.253 